\title{
Women associated vaginal symptoms with disease and sexual infidelity
}

Karasz A, Anderson M. The vaginitis monologues: women's experiences of vaginal complaints in a primary care setting. Soc Sci Med 2003;56:1013-21.

\section{$Q$ How do women interpret vaginal sensations as symptoms and construct these symptoms as problems requiring medical care?}

\section{DESIGN}

Qualitative study.

\section{SETTING}

A family health centre serving a multi-ethnic, working class population in New York City, USA.

\section{PARTICIPANTS}

44 women $>18$ years of age who were diagnosed with vaginitis (ICD9 code 616.10 ) in the previous 4 months.

\section{METHODS}

Women participated in semistructured telephone interviews of 30-45 minutes, during which interviewers took indepth notes. Data collection and analysis were based on an iterative process. Women were asked to generate illness narratives of symptom and treatment experiences. The semistructured component of the interview was based on the Illness Representation Model.

\section{MAIN FINDINGS}

Most women had some combination of itching, discharge, and odour. Conceptions of [a] normal [vagina]. Women judged normality based on characteristics of vaginal discharge (eg, quantity, colour, odour, consistency, and timing in relation to the menstrual cycle). Although most felt that some discharge was normal, one fifth thought that a normal vagina should be dry and odour free. Causation. About half of the women thought that their symptoms resulted from infectious causes. Many did not distinguish between vaginitis and sexually transmitted diseases (STDs). Some women worried that their symptoms were caused by cancer or previous sexual misbehaviour. Consequences. Many women thought their symptoms were "somewhat serious" or "very serious." Although most women had not discussed these concerns with their healthcare providers, many worried that if not treated, the infection could spread and cause sterility or even death. Missed days of work or school were common. Women worried that others could smell their vaginal odour; some showered, douched, or changed underwear several times each day. About one quarter of women reported sexual problems because of their symptoms (dyspareunia or concern about passing infection to partners). Treatment and management. Most women sought medical attention after other options had failed. About one third used an over the counter yeast infection medication, which was often left over from previous infections. Some bathed with special soaps. Douching Bronx, NY, USA. akkarasz@montefiore.org Source of funding: no external funding. was commonly done to feel "fresh and clean," particularly after menstrual periods, as menstrual blood was thought to be inherently dirty. Treatment outcomes. Of 31 women who reported the outcome of medical consultations, 24 had symptom remission within a few days, 4 improved but subsequently relapsed, and 4 did not improve. Women with chronic symptoms were often dissatisfied with treatment outcome and wanted a "permanent cure." Stigma and disclosure. Rigorous hygienic practices were often related to feelings of disgust and shame about vaginal symptoms. Stigma also arose from an assumption that vaginal symptoms were caused by sex, and thus might be seen as evidence of promiscuity. Women were often reluctant to discuss vaginal symptoms with partners, fearing confrontations or accusations about their partner's or their own infidelities.

\section{CONCLUSIONS}

Within a primary care context, women associated vaginal symptoms with disease. These symptoms were thought to be caused by sexually transmitted diseases, and thus were evidence of sexual infidelity.

\section{Commentary}

I's hard to believe, but before 1970, little information on women's health or women's bodies was available to women. What little women knew about their bodies was often treated as a secret or meted out by physicians and other healthcare professionals according to what they believed women should know. Generations of young women, myself included, learned about normal female genitalia, menstruation, and reproduction from pamphlets or the school nurse. Typically these episodes of teaching, which sometimes included a film, occurred with our male classmates removed, the lights dimmed, and the blinds down. This encouraged a sense of shame and secrecy about our normal functions.

Since the advent of the landmark Our Bodies, Ourselves by the Boston Women's Health Collective, books on women and women's health have proliferated. Women are encouraged to screen themselves for cervical cancer and to protect themselves against sexually transmitted diseases. Advertisers market feminine hygiene products to women to cover the "nasty" smells we have. Sanitary products are promoted to help us feel "fresh" and "clean."

The study by Karasz and Anderson confirms that many women do believe their bodies aren't clean and that any vaginal symptoms, normal or not, are symptoms of something seriously wrong or are related to sex and therefore immoral. For nurses who work in women's health, this study is a reminder that we need to help women to understand that menstruation and sexual activity are normal and healthy and to identify the differences between normal, healthy vaginal discharge and that which is indicative of disease.

enore Riddell, RN, MSN BC Women's Hospital Vancouver, British Columbia, Canada 